Relations industrielles

Industrial Relations

\title{
Blouin, Rodrigue et Fernand Morin, Droit de l'arbitrage de grief
}

\section{Claudette Ross}

Volume 50, numéro 4, 1995

L'ergonomie et les relations industrielles

Ergonomics and Industrial Relations

URI : https://id.erudit.org/iderudit/051060ar

DOI : https://doi.org/10.7202/051060ar

Aller au sommaire du numéro

Éditeur(s)

Département des relations industrielles de l'Université Laval

\section{ISSN}

0034-379X (imprimé)

1703-8138 (numérique)

Découvrir la revue

Citer ce compte rendu

Ross, C. (1995). Compte rendu de [Blouin, Rodrigue et Fernand Morin, Droit de l'arbitrage de grief]. Relations industrielles / Industrial Relations, 50(4), 887-890. https://doi.org/10.7202/051060ar

Tous droits réservés (C) Département des relations industrielles de l'Université Laval, 1995
Ce document est protégé par la loi sur le droit d'auteur. L’utilisation des services d'Érudit (y compris la reproduction) est assujettie à sa politique d'utilisation que vous pouvez consulter en ligne.

https://apropos.erudit.org/fr/usagers/politique-dutilisation/ 
run in nature. Therefore, from the shareholders must come a willingness to lengthen the time horizon for results.

From unions or other forms of worker representation must come a willingness to cooperate with management to bring about the needed changes to improve productivity and, thus, better compete in the global economy. Examples are given of such cooperation and include, among others, the United Auto Workers and GM at NUMMI and Saturn; the Communication Workers of America and AT\&T; as well as various efforts involving the United Steelworkers, and the Amalgamated Clothing and Textile Workers Union. As the authors appropriately point out, such union cooperation is impossible without the willingness of management to provide worker "voice" and job security.

From the government must come support for an improved employment and training policy, as well as a reconstitution of our labour laws and industrial relations framework. In the latter instance, it is the authors' contention that current U.S. labour law which often promotes adversity between the parties, should be changed to encourage cooperation.

Lastly, to facilitate the advancement of the above changes, the authors suggest that the academics involved in this area of study need to reconstruct the multidisciplinary networks envisioned some half century ago when the Industrial Relations Research Association was first organized. Indeed, one can't help noting the authors' disdain for the overly technical and narrow orientation of current teaching and research in this area.

As a believer in the need for cooperative labour-management relations, this reviewer is in agreement with both the analysis and prescription for success. "On the other hand," as we economists are prone to say, providing the prescription won't make it so. Convincing both labour and management to relinquish various prerogatives and to embrace the necessary trust in their counterpart is only the beginning. All parties to the relationship will need convince the nay sayers in their respective organizations of the need to cooperate and of the necessity of some short run costs to allow for long run success. As experience has indicated, this will not be easy. Lastly, the mid term elections in the U.S. would not give one much optimism that the government is likely to play the prescribed role. Nevertheless, as Kochan and Osterman indicate, unless we as a nation of vested interests are willing to sit down and work cooperatively with each other, the prospects for the future leave much to be desired.

MARTIN M. PERLINE Wichita State University

\section{Droit de l'arbitrage de grief}

par Rodrigue BLOUIN et Fernand MORIN, Cowansville, Les Éditions Yvon Blais, 1994, 694 p., ISBN 2-89073-977-5.

Il s'agit de la $4^{\mathrm{e}}$ édition de cet ouvrage, publié pour la première fois en 1975. L'édition précédente portait le titre de Arbitrage des griefs 1986. Le nouveau titre apparait plus éclairant, puisqu'il s'agit bien, comme l'indiquent les auteurs dans l'avant-propos, d'un ouvrage sur le droit de l'arbitrage de grief et non sur l'interprétation et l'application du contenu de la convention collective par l'arbitre. Cet ouvrage insiste sur les aspects institutionnels de l'arbitrage de grief dans le contexte du régime général des rapports collectifs du travail ; les règles propres à certains secteurs particuliers et à certains secteurs dérogatoires y sont étudiées sur une base comparative et à titre complémentaire. L'objectif 
des auteurs est demeuré le même, soit d'aider ceux qui veulent comprendre ce mécanisme de justice, sa nature et sa portée pour qu'ils puissent, seuls ou avec l'aide de conseillers, en faire un meilleur usage.

Comme l'expliquent les auteurs, la réédition de cet ouvrage était devenue nécessaire en raison des changements importants ayant marqué le régime d'arbitrage de grief et de la modification des comportements des parties, des arbitres et des tribunaux de contrôle au cours de la dernière décennie. La nouvelle édition fait donc état des modifications législatives qui ont soit élargi, soit restreint le champ d'action des arbitres et elle fait une large place à la jurisprudence arbitrale et à la doctrine récentes. Pour connaitre les devoirs et les pouvoirs des arbitres de grief, les auteurs s'appuient également, et à juste titre, compte tenu de leur propos, sur la jurisprudence des tribunaux de droit commun.

Cette nécessaire mise à jour a été effectuée en conservant la structure générale de l'édition précédente et sa division en dix titres. À l'intérieur de chacun des titres, une attention particulière a été accordée à la subdivision en chapitres, sections et sous-sections et à l'intitulé de ceux-ci de façon à refléter davantage les lignes de force et d'intérêt du texte.

Les quatre premiers titres abordent les coordonnées générales du régime d'arbitrage de grief. Le titre I est consacré au profil historique et à l'apport de l'arbitrage. Les auteurs y retracent l'évolution de l'implantation du régime, situent le régime dans le cadre plus général des rapports collectifs du travail et dégagent le particularisme de la fonction arbitrale. La nouvelle édition permet de mieux saisir l'évolution de l'action législative en accordant plus de place aux diverses lois et en faisant ressortir plus clairement encore les grandes lignes de cette évolution.
Le titre Il traite des sources juridiques de l'aménagement et du fonctionnement du régime. Après avoir posé la toile de fond juridique du régime, les auteurs présentent ces différentes sources juridiques, soit la législation habilitante, la convention collective, la pratique et la jurisprudence. Au sujet des ajouts ou modifications à ce titre, on remarque notamment l'ajout d'une section sur l'apport relatif des diverses sources juridiques, qui traite entre autres de l'influence des chartes et du nouveau Code civil du Québec. Les chapitres sur la pratique et la jurisprudence ont été revus et simplifiés.

Le titre III porte sur la notion de grief et sur les parties à l'arbitrage. Les auteurs y précisent également le particularisme des griefs assimilés par voie législative.

Le titre IV traite de la fonction arbitrale et de son titulaire, en dégageant les caractéristiques fondamentales de cette fonction, le statut de ses titulaires ainsi que leurs pouvoirs et devoirs. Les auteurs font davantage ressortir dans cette nouvelle édition le fait que l'arbitre exerce les fonctions d'un tribunal quasi-judiciaire et constitue de ce fait un tribunal au sens de la Charte des droits et libertés de la personne.

Le titre $V$ présente la procédure préalable et les préparatifs à l'arbitrage en insistant davantage sur les aspects pratiques de ces préparatifs. En outre, des sections spécifiques sont maintenant consacrées à la computation des délais et à l'assignation des témoins.

Les deux titres suivants sont consacrés au déroulement même de l'arbitrage. Le titre VI traite des objections préliminaires, de leur nature, de leur objet, de la procédure de soumission d'une objection et de la décision sur une objection. Sur le plan conceptuel, les auteurs clarifient les distinctions existant entre les différents moyens préliminaires. Leur souci de mettre davantage l'accent sur certains aspects pratiques du processus se manifeste ici aussi par 
l'ajout de sections sur la preuve à apporter en cette matière et sur le pourvoi à l'encontre d'une décision préliminaire. Le titre VII pour sa part porte sur l'enquête proprement dite, ses coordonnées générales, la preuve et les plaidoiries. La notion de pertinence de la preuve $y$ reçoit une attention particulière. Une section a également été ajoutée sur la preuve extrinsèque.

Les fonctions principales de l'arbitre de grief, soit l'interprétation et l'application de la convention collective, sont abordées dans les deux titres suivants. Le titre relatif à l'interprétation de la convention collective (titre VIII) a été remanié en profondeur et sa rédaction resserrée, ce qui le rend plus facile d'accès. On présente toujours cependant les différentes règles utiles pour l'interprétation de la convention collective. Le titre consacré à l'application de la convention collective (titre IX) traite des aspects institutionnels et des aspects pratiques de ce contrōle. La section relative aux pouvoirs généraux de contrôle et de correction s'est notamment enrichie de développements sur les ordonnances de sauvegarde, sur le pouvoir d'accorder des dommages-intérêts et sur les effets fiscaux du paiement des sommes dues.

La décision arbitrale fait l'objet du dernier titre (titre $X$ ). Les auteurs $y$ traitent du processus décisionnel et des effets de la décision. La section relative au contrôle judiciaire a été remaniée pour tenir compte des modifications apportées en jurisprudence dans la façon de déterminer ce qui est pour l'arbitre une question juridictionnelle ou intrajuridictionnelle.

Un tableau des taux d'intérêts, une bibliographie, un index de la jurisprudence citée, un index des articles du Code du travail ont été ajoutés à cette nouvelle édition. La présentation graphique de l'index analytique a également été aérée, ce qui le rend beaucoup plus facile à consulter. Il aurait cependant pu être utile pour les praticiens de préciser les limites temporelles de la mise à jour de la jurisprudence.

Cette nouvelle édition ne se limite donc pas à une simple mise à jour de l'édition antérieure. Des ajouts y ont été apportés et certaines parties du texte ont été remaniées et épurées, le tout dans le respect de l'objectif des auteurs: permettre au lecteur non seulement de connaître les divers éléments du régime de l'arbitrage des griefs mais également de comprendre les finalités de ce régime et les liens existant entre ces divers éléments.

La grande qualité de cet ouvrage réside en effet dans cette approche intégrée et intégrative ainsi que dans la vision critique adoptée par ses auteurs. Ceux-ci ne se contentent pas de rapporter et d'expliquer le droit positif de façon impersonnelle, mais font véritablement ouvre de doctrine en exposant leur conception de ce que devrait être le régime d'arbitrage de grief.

On peut ne pas être toujours d'accord avec certaines des prises de positions spécifiques des auteurs et constater qu'ils n'échappent pas toujours au formalisme qu'eux-mêmes dénoncent (particulièrement en ce qui a trait à la façon de constater un règlement intervenu entre les parties). On peut également regretter leur laconisme sur certaines questions (notamment celles de la médiation pré-arbitrale, de l'arbitrage accéléré et de la médiation préventive qui ne sont que brièvement abordées et celle de l'estoppel, dont on peut se surprendre qu'elle ne fasse l'objet que d'une note infrapaginale). De même, l'approche positive adoptée par les auteurs dans la rédaction de l'ouvrage (dont on ne peut douter qu'elle soit volontaire compte tenu de leurs commentaires sur la facture de la décision arbitrale) peut avoir pour effet de masquer au lecteur non averti les principales critiques adressées au régime d'arbitrage de grief.

La conception du régime d'arbitrage de grief proposée par les auteurs et qui 
se dégage en filigrane dans tout leur ouvrage constitue toutefois une avenue à privilégier pour remédier à certaines des limites actuelles du régime: les auteurs s'élèvent contre une trop grande judiciarisation du régime et contre l'importation de concepts et de techniques inappropriés de droit civil ou de common law, l'arbitrage de grief devrait selon eux se détacher des approches traditionnelles et civilistes de la convention collective, pour la comprendre et la saisir d'une manière plus concrète, plus souple et plus adaptée aux besoins des parties, en privilégiant une approche systémique, à l'opposé des exégèses.

En conclusion, on ne peut donc que souligner le caractère indispensable de cet ouvrage pour tous les praticiens de l'arbitrage de grief et sa contribution majeure à la doctrine.

ClaudeTte RosS

Université du Québec à Montréal

\section{Le droit des salariés à la négociation collective : principe général du droit} par Marie-Laure MORIN, Paris, L.G.D.J., 1994, 681 p., ISBN 2-275-00420-3 et ISSN 0520-0180.

Version actualisée d'une thèse de doctorat, l'imposant ouvrage de madame Morin, chargée de recherche, CNRS, au Centre d'études juridiques et économiques de l'emploi de l'Université de sciences sociales de Toulouse, présente le droit français de la négociation collective, non pas d'une façon linéaire, mais à travers la démonstration de sa nature essentielle. Son titre révèle simplement le principe qui irradie ce droit: un véritable droit des salariés à la négociation collective, principe général du droit. Ce droit des salariés "à la négociation collective de l'ensemble de leurs conditions de travail et d'emploi et de leurs garanties sociales ", qu'affirme la Loi du 13 juillet 1971 , se retrouve aujourd'hui en l'article L. 131-1 du Code du travail, à la tête de l'ensemble de ses règles d'exercice ; il traduit l'affirmation constitutionnelle de 1946 (alinéa 8 du Préambule de la Constitution): "Tout travailleur participe par l'intermédiaire de ses délégués à la négociation collective des conditions de travail ainsi qu'à la gestion des entreprises ".

Il s'agit bien là d'un principe général du droit, notion dont l'auteure établit préalablement et théoriquement les exigences et la portée. Par sa place hiérarchique, par sa généralité d'extension, un tel principe "assure la cohérence des institutions positives qui le matérialisent et l'évolution du système juridique dans son environnement (naissance de nouvelles normes, transformation interne du système juridique, etc.)" (p. 58).

Conforté par l'évolution du droit international (cf. les conventions $\mathrm{n}^{\circ} 87$ et 98 de l'OIT notamment et le développement plus récent du droit européen), l'actuel droit des salariés à la négociation collective est édifié en droit interne, comme le démontre la première partie de l'ouvrage, sur l'affirmation progressive de la négociation collective : conception d'abord contractuelle de la négociation collective (loi du 25 mars 1919), régime de la convention étendue de la loi de 1936, qui fait de la branche, de la profession, le niveau central de négociation, reconnaissance de la capacité normative des parties à différents niveaux de négociation résultant de la loi de 1950, généralisation de l'autonomie collective, non pas au sens absolu du droit allemand, mais sous l'égide de la loi. Ce droit de négocier qu'affirme la loi de 1971 est donc devenu un droit général de négocier : accords d'entreprises, conventions nationales locales ou régionales, de portée sectorielle, ou même accords interprofessionnels. Centré jusque-là sur le négocié, droit de l'accord, le droit à la négociation allait par la 